\title{
Comparison of Olfactory Cleft Width and Volumes in Patients with COVID-19 Anosmia and COVID-19 Cases Without Anosmia
}

\author{
Deniz Esin Tekcan Sanlia Aytug Altundag ${ }^{b}$ Duzgun Yıldırım ${ }^{c}$ \\ Sedat Giray Kandemirlid Ahmet Necati Sanlie

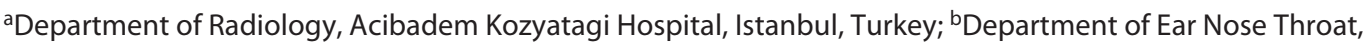 \\ Acibadem Taksim Hospital, Istanbul, Turkey; ' Department of Radiology, Acibadem Taksim Hospital, Istanbul, Turkey; \\ ${ }^{\mathrm{d}}$ Department of Radiology, The University of lowa, lowa, IA, USA; 'Department of General Surgery, Cerrahpasa \\ Medical Faculty, Istanbul University-Cerrahpasa, Istanbul, Turkey
}

\section{Keywords}

COVID-19 · Anosmia - Olfactory cleft - Olfactory cleft volume . Olfactory cleft width . Paranasal computed tomography . Threshold-discrimination-identification · Age

\begin{abstract}
Introduction: The aim of this study was to assess the relationship between olfactory cleft width/volume and COVID19-related anosmia. Methods: This study consisted of PCR-proven COVID-19 patients. Cases with COVID-19-related anosmia constituted Group 1 and cases without any olfactory dysfunction (OD) throughout COVID-19 infection or after recovery constituted Group 2. A total of 50 patients were included in the study, comprising 24 cases in Group 1 and 26 cases in Group 2. Group 1 patients underwent a 4-item-odor identification test during active symptoms and a Sniffin' Sticks test after reconversion of PCR results to negative. All patients in Group 2 also underwent the Sniffin' Stick test to document normosmia. All cases had paranasal sinus CT performed. Olfactory cleft widths and olfactory volumes were measured. The differences in width and volume between groups and the correlation with odor test scores (threshold-discrimination-identification [TDI]) were calculated. In addition, regression analyzes analysis was performed
\end{abstract}

karger@karger.com www.karger.com/orl

(c) 2021 S. Karger AG, Basel

Karger! for cleft widths, volumes, and TDI scores according to age. Results: Olfactory cleft widths and olfactory volumes were significantly higher in Group 1 than those in Group 2 ( $p=$ $0.001 ; p<0.01$ ). There was a significant negative correlation between total TDI scores and olfactory cleft widths and total olfactory volumes ( $r=-0.665 ; r=-0.731$, respectively). Patients younger than 40 years of age had significantly higher right olfactory cleft width, left olfactory cleft width, and olfactory cleft volume than those in patients older than 40 years of age ( $p=0.004, p=0.005, p=0.003 ; p<0,01$, respectively). However, patients younger than 40 years of age had a significantly lower total TDI score and in all other values individually $(\mathrm{t}-\mathrm{d}-\mathrm{i})$ than those in patients older than 40 years of age $(p=$ $0.004 ; p<0.01)$. Conclusion: Patients with COVID-19-related OD had larger olfactory cleft width and volumes than those without OD in this study. Total TDI score was found to be inversely correlated with cleft width and volume.

(c) 2021 S. Karger AG, Basel

\section{Introduction}

There have been a substantial number of manuscripts on clinical symptoms of COVID-19 since the start of the pandemic. One of the most commonly reported symptoms 
besides the upper respiratory tract infection is olfactory dysfunction (OD) [1]. Though there is variability in OD rates ranging between 5 and $85 \%$ among different ethnicities, higher incidence in the younger patient population with female predominance has been noted [2-6]. OD has important diagnostic implications as it may be the only symptom of the infection $[7,8]$. Clinicians should have a high level of suspicion for COVID-19 infection in cases with sudden onset of OD without underlying etiological factors. Additionally, more recent studies showed a relatively milder COVID-19 disease course in patients with anosmia $[1,9]$. In this aspect, COVID-19 anosmia has prognostic predictive value as well. However, severe patients may just not be able to answer the question of OD nor notice the symptoms.

The most important distinguishing feature of $\mathrm{CO}$ VID-19 anosmia compared to OD related to other upper respiratory viruses is a relatively low association with nasal discharge/obstruction, sudden onset of findings, and spontaneous recovery in the majority of cases without sequela $[3,10,11]$. Though several articles are discussing potential etiopathogenesis, there is currently no widely accepted hypothesis.

Recently, several studies have focused on OC and post-viral anosmia [12, 13]. Increasing cleft width could increase viral exposure load and result in a higher receptor occupancy [12-14]. Additionally, Alzheimer disease, in which viral pathogenesis hypotheses are entertained, has a high rate of OD $[15,16]$. In this regard, cleft width can have important cause and effect relation and given the high rate of COVID-19 anosmia, we tried to quantitatively assess the relation between olfactory cleft volume and COVID-19 anosmia. Thus, in this study, we assessed olfactory cleft widths and volumes in COVID-19 patients both with anosmia and without OD in order to get a better understanding of COVID-19 anosmia. We showed that patients with COVID-19 associated anosmia had greater cleft widths and volumes.

\section{Materials and Methods}

\section{Study Group}

This study included patients diagnosed with COVID-19 infection between March and July 2020. All included cases had PCR confirmed diagnosis from nasopharyngeal swab samples. Cases were classified into 2 groups based on the presence of OD during the active disease course or at the recovery stage: Group 1: COVID-19 patients with OD and Group 2: COVID-19 patients without any OD. OD was defined as changes in the form of hyposmia, cacosmia, and anosmia that patients noticed compared to their olfactory status before the disease, and it was also objectively shown by objective olfactory tests. Patients with a complaint of OD but not found to be dysfunctional on objective olfactory tests were not included in Group 1. All cases in Group 1 had a dedicated paranasal sinus CT to assess for underlying organic or obstructive pathologies. In order to minimize the radiation exposure, we have included patients with prior paranasal sinus CTs for the control group. This prior CTs were acquired before the COVID-19 pandemic between January 2017 and December 2019. Cases with studies older than 2 years have not been included to minimize the possible time-related changes. We selected cases that underwent CT for indications like tinnitus or headache that had not been shown to have a relation to olfactory cleft or olfactory function so far. We have excluded patients with nasal septal deviation or pathologically hypertrophied turbinates since such findings could affect the airflow dynamics in the nasal cavity. A total of 50 patients were included in the study, comprising 24 cases in Group 1 and 26 cases in Group 2.

\section{Inclusion and Exclusion Criteria}

All cases in both groups were PCR confirmed COVID-19 patients. Patients with a past medical history of chronic rhinosinusitis, nasal polyposis, allergic or idiopathic rhinitis, post-traumatic olfactory loss, malignant tumors, and/or a history of oncology treatment, a history of nasal or paranasal surgery, and pregnancy were excluded for both groups. Also, patients with nasal septal deviation or pathologically hypertrophied turbinates that could affect the airflow dynamics in the nasal cavity have been excluded in both groups. Patients with COVID-19 infection and OD who were determined to have normosmia (a threshold-discrimination-identification score [TDI] score of $>30.5$ ) on the Sniffin' Stick test were included in Group 2.

\section{Olfactory Examination}

In order to objectively assess the OD, all patients in Group 1 had a 4-item-odor discrimination test during the active infection course and the Sniffin' Stick test after conversion of PCR results to negative. All patients in Group 2 also underwent the Sniffin' Stick test to document normosmia. All patients in Group 1 were found to be anosmic (TDI score $<16.5$ ) according to the olfactory testing, while all Group 2 patients were normosmic (TDI score >30.5) [17-20].

\section{4-Item-Odor Identification Test}

OD in COVID-19 patients was initially assessed using a 4-itemodor identification test. The test consists of 4 bottoms including rose, clove, orange, and mint. Using a multiple-choice paradigm, patients were asked to find the correct odor descriptions from a verbal list of 4 descriptors each. A score of 0 was accepted as anosmia in 4 odor identification test. This test was applied only to Group 1.

\section{Sniffin' Sticks Olfactory Test}

Olfactory testing was performed using the Sniffin' Sticks test (Burghart, Wedel, Germany) [17-20]. The mean interval between Sniffin' Sticks tests and the onset of anosmia complaints was 21.75 \pm 4.58 days (14-30 days) in Group 1 . The mean interval between Sniffin' Sticks tests and COVID-19 diagnosis was $23.42 \pm 4.68$ days (15-30) in Group 2.

During the test, odorants were presented in felt tip pens. For odor presentation, the investigator first removed the cap and then placed the tip of the pen in front of the subject's nostrils. With this test 

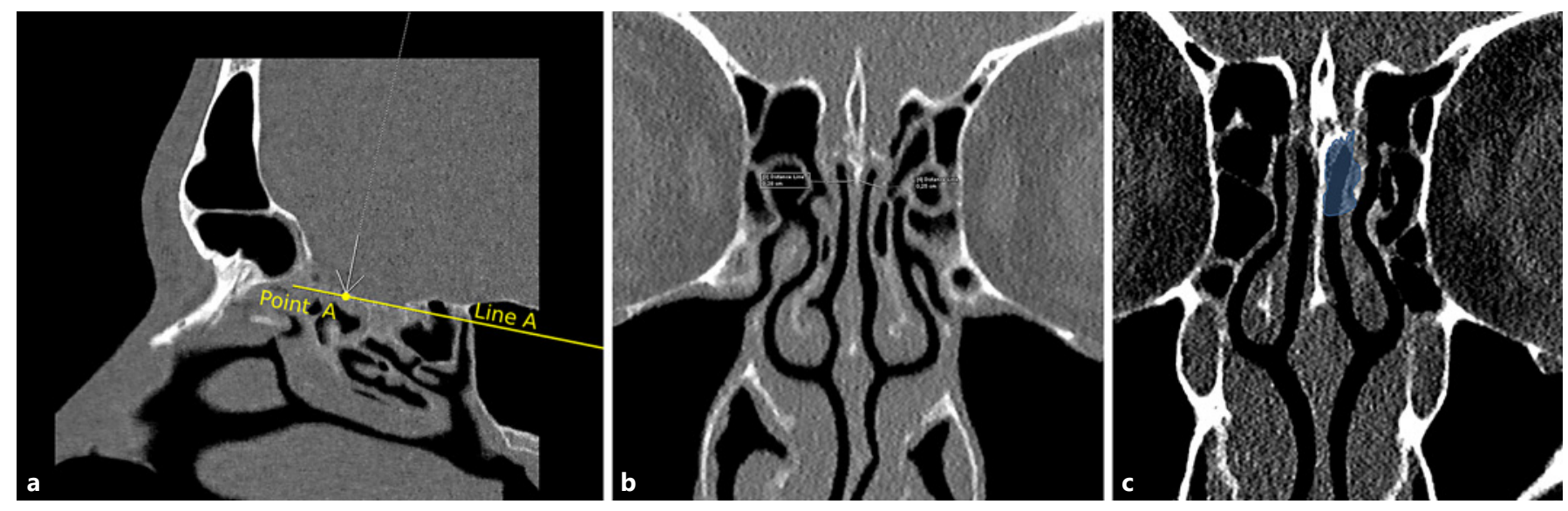

Fig. 1. Cleft measurements were calculated at the junction of anterior $1 / 3$ and posterior $2 / 3$ because of more stable anatomy with preserved aeration and symmetric bilateral clefts at this point. More anterior or posterior slices had alterations in the anatomy and asymmetry (partial cleft opacification, medialization/lateralization of laminae). This may not have importance in daily clinical practice; however, measurement of a small region like the olfactory cleft required a standard methodology. a In order to measure the olfactory cleft width, a line (line A) parallel to the cribriform plate is defined along the olfactory cleft borders on paranasal sinus $\mathrm{CT}$ images in sagittal reconstruction. The intersection of anterior $1 / 3$ and posterior $2 / 3$ of this line is marked (point A). b Coronal oblique reformats are created perpendicular to line $\mathrm{A}$, and olfactory cleft widths are measured at the intersection of anterior $1 / 3$ and posterior $2 / 3$ of this line (point $\mathrm{A}$ ) and $10 \mathrm{~mm}$ inferior to the cribriform plate. During olfactory cleft width measurement, air and mucosa are included, whereas bone and cartilaginous structures are excluded. c In the coronal plan image (same as b) created in the soft tissue window, a sample of olfactory cleft volume measurement (marked as blue highlighted region) is shown by drawing the contours of the osseous cortex (including the cleft mucosa) taking into account a $1 \mathrm{~cm}$ depth of the cribriform plate. Bone (thick arrow) and cartilaginous structures (arrow at nasal septum) are not included in the total volume. battery, the olfactory function was examined bilaterally in terms of the odor threshold (T), discrimination (D), and identification (I). The clinical evaluation of olfactory performance was based upon a composite of the TDI score represented by the sum of the scores from 3 subtests. A TDI score below 16.5 was accepted as functional anosmia [17-20]. The applicability of the "Sniffin" Sticks" test for the target population has been previously validated [21].

\section{Imaging}

All Group 1 cases included in the study had paranasal sinus CT including the paranasal sinuses available at the time of olfactory evaluation. All CT exams were performed with a $128 \times 2$-slice dual-source CT scanner (Siemens, Flash Definition, Erlangen, Germany). After screening of paranasal sinus region using $0.625 \mathrm{~mm}$ collimation, the OC region was reformatted at $0.4 \mathrm{~mm}$ sectionthickness and $0.1 \mathrm{~mm}$ increment with a centralized smaller FOV. Mucosal, secretory and inflammatory changes, transient opacifications could mislead the true intranasal volume. To overcome this in a standard fashion, aeration of OC was assessed by creating sharp-edge (bone kernels) reconstructions on the coronal plane. The CT scans were analyzed for olfactory cleft width and volumes. In both groups, the olfactory cleft width was measured for both right and left sides separately, and mean values were calculated for statistical analysis. CT measurements were performed with a special workstation that allows very precise digital measurements (Syngo.Via Software [VB30A, Siemens], Siemens, Erlangen, Germany). The boundaries of the olfactory cleft were determined using successive coronal plane sections of $1 \mathrm{~mm}$. Same regions and landmarks that have been defined in our prior publications were used for standardization and optimization $[12,14]$. The anterior boundary was defined as the anterior attachment of the middle turbinate and the posterior boundary as the anterior wall of the sphenoid sinus. The medial boundary was the nasal septum, and the lateral boundary was the middle and superior turbinates [12, $14,22]$. On CT, OC diameters were measured on soft tissue windows at coronal and axial planes by including mucosa and adjacent air density structures. Bone and cartilaginous structures were not included. For this reason, measurements of right and left olfactory clefts were performed separately and subsequently mean value was calculated. The volume measurements were calculated in the coronal plane perpendicular to the horizontal plane parallel to the cribriform plate at the intersection point of anterior $1 / 3$ and posterior $2 / 3$ (Fig. 1a), which could be evaluated more easily since the aeration was always preserved (Fig. 1b). In this coronal section, perpendicular to a plane parallel to the cribriform platform, a region up to $10 \mathrm{~mm}$ deep from the roof of the cleft was taken into account and appropriate voxel of interest plans was measured by making manual-free voxel of interest drawings (Fig. 1c). A greater depth could result in alteration of configuration due to angulation of middle concha laminae. This may hamper a standardized measurement. A shallower depth may have excluded a great surface lined with olfactory epithelium. We determined the cutoff depth as 10 $\mathrm{mm}$ after looking at all cases prior to measurements. For more standardized results, automatic calculations were made using a computer-aided software program (Multimodality Reading Volume Measurement) with manual adjustments in coronal (Fig. 2a) and axial planes (Fig. 2b) instead of measuring 1 by 1 olfactory cleft width, depth, and length in the volume calculation. Calculated volumes 

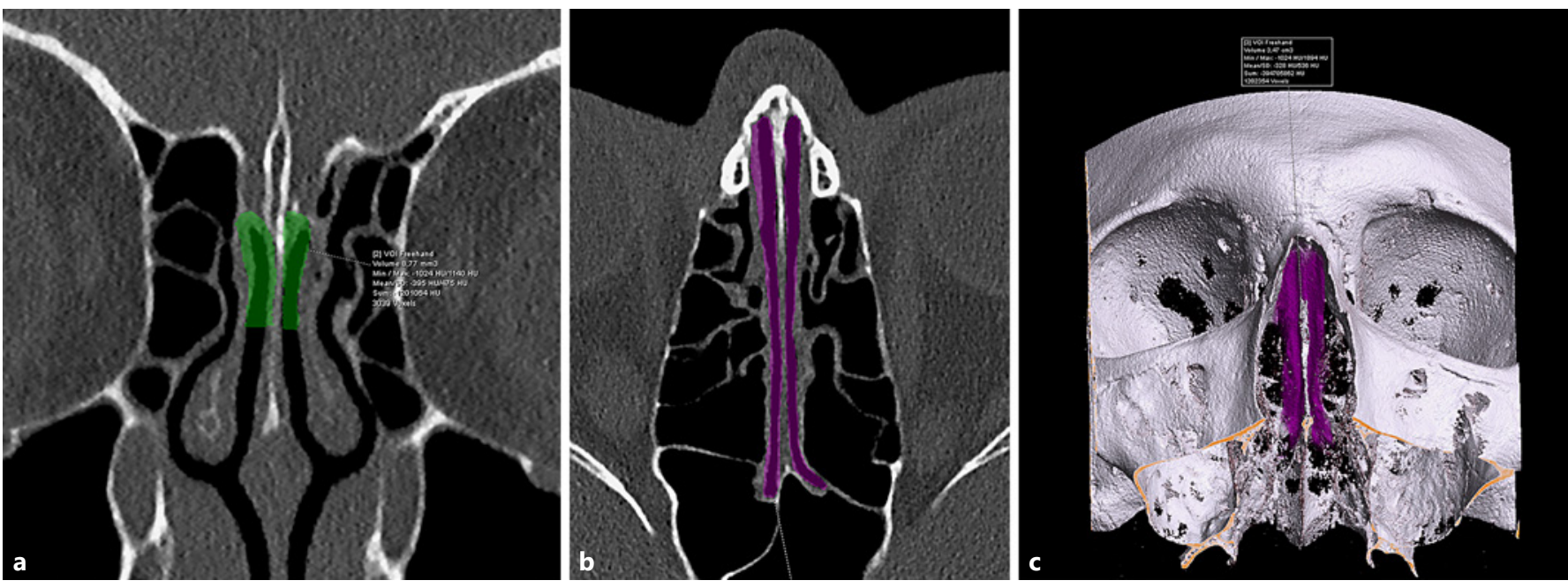

Fig. 2. a Measurement of right and left olfactory cleft volumes on coronal plane. During segmentation, structures with air and soft tissue density are included. b Measurement of right and left olfactory cleft volumes on axial plane, depicting the anterior and posterior margins of olfactory cleft. c 3D volumetric images illustrate right and left olfactory cleft volume measurements and mean density of volume of interest.

were recorded in $\mathrm{mm}^{3}$, and the average density of the total volume within the voxel was recorded in Hounsfield Unit (Fig. 2c).

All measurements were performed by a single radiologist blind to the demographic and clinical information of the patients for standardization. The radiologist made 2 measurements per patient for all parameters, and the arithmetic mean of measurements was used for statistical analysis.

\section{Ethics}

The study protocol was approved by the Medical Research Ethics Committee of Acibadem University (decision No: 2020-13/6, date. June 25,2020$)$. The study protocol conforms to the ethical guidelines of the 1975 Declaration of Helsinki as reflected in a priori approval by the institution's human research committee.

\section{Statistical Analysis}

Number Cruncher Statistical System software was used for statistical analysis. Parametric data are presented as mean and standard deviation and nonparametric data as median and range. The Shapiro-Wilk test was used to assess the normal distribution of the data. Student's $t$ test and Mann-Whitney U tests were used to assess differences between groups for normally distributed variables and nonparametric variables, respectively. Spearman correlation test was used to assess the relation between TDI scores and olfactory cleft measurements. Statistical significance is accepted as $p<0.05$. Backward regression analysis was performed to assess the effect of age, right cleft width, left cleft width, and cleft volume on TDI.

\section{Results}

The mean age of the study population was $43.96 \pm$ 16.32 (range: 19-90) years. Cases in Group 1 (35.33 \pm $10.50)$ were younger than those in Group $2(51.92 \pm 16.84)$
( $p=0.001)$. The mean time between the CT scan times of the patients and the COVID-19 infection was $83.95 \pm$ 28.03 (39-125) days in Group 1 and $20.61 \pm 9.14(4-34)$ months in Group 2.

All cases in Group 1 had a score of 0 on the 4-item-odor identification test, consistent with anosmia. The Sniffin' Sticks test results in Group 1 had a mean T-score of 1.27, D-score of $0.87, I$-score of 1.46, and a TDI score of 3.60 . Group 2 patients had a mean T-score of 12.5, D-score of 12.92, I-score of 12.80, and total TDI score of 38.23. Group 1 patients had significantly lower TDI scores, and based on results, all cases in Group 1 were anosmic, and all cases in Group 2 were normosmic $(p<0.001)$.

Mean olfactory cleft widths in Group 1 patients $(2.92$ $\pm 0.47 \mathrm{~mm}$ ) were significantly larger than those in Group 2 patients $(1.95 \pm 0.27 \mathrm{~mm})(p=0.001)$ (Table 1). Similarly, total olfactory volumes were significantly larger in Group $1\left(340.04 \pm 53.09 \mathrm{~mm}^{3}\right)$ than Group 2 (161.19 \pm $\left.19.70 \mathrm{~mm}^{3}\right)(p=0.001)$ (Table 1).

Linear regression analysis for OD based on age was carried out. Patients younger than 40 years of age had significantly higher right olfactory cleft width, left olfactory cleft width, and olfactory cleft volume than those in patients older than 40 years of age $(p=0.004, p=0.005, p=$ $0.003 ; p<0.01$, respectively). However, patients younger than 40 years of age had a significantly lower total TDI score and in all other values individually $(\mathrm{t}-\mathrm{d}-\mathrm{i})$ than those in patients older than 40 years of age $(p=0.004 ; p<0.01)$ (Table 2).
Tekcan Sanli/Altundag/Yıldırım/ Kandemirli/Sanli 
Table 1. Paranasal CT features and Sniffin' stick test results in COVID-19 patients grouped based on the presence of OD

\begin{tabular}{|c|c|c|c|c|}
\hline & \multicolumn{4}{|l|}{ Anosmia } \\
\hline & total & present $(n=24)$ group 1 & absent $(n=26)$ group 2 & $p$ value \\
\hline \multicolumn{5}{|l|}{ Age } \\
\hline $\begin{array}{l}\text { Mean } \pm \text { SD } \\
\text { Mean olfactory cleft width, } \mathrm{mm}\end{array}$ & $43.96 \pm 16.32$ & $35.33 \pm 10.50$ & $51.92 \pm 16.84$ & $0.001^{\mathrm{a}, * *}$ \\
\hline $\begin{array}{l}\text { Mean } \pm \text { SD } \\
\text { Olfactory cleft volume, } \mathrm{cm}^{3}\end{array}$ & $2.41 \pm 0.62$ & $2.92 \pm 0.47$ & $1.95 \pm 0.27$ & $0.001^{a, * *}$ \\
\hline Mean \pm SD & $247.04 \pm 98.33$ & $340.04 \pm 53.09$ & $161.19 \pm 19.70$ & $0.001^{\mathrm{a}, * *}$ \\
\hline $\begin{array}{l}\text { OD, olfactory dysfunction. } \\
\text { a Student } t \text { test. } \\
\text { ** } p<0.01 \text {. }\end{array}$ & & & & \\
\hline
\end{tabular}

Table 2. Comparison of cleft width volumes and TDI scores according to age

\begin{tabular}{|c|c|c|c|}
\hline & \multicolumn{2}{|l|}{ Age } & \multirow[t]{2}{*}{$p$ value } \\
\hline & $<40$ years $(n=22)$ & $\geq 40$ years $(n=28)$ & \\
\hline \multicolumn{4}{|c|}{ Right olfactory cleft width, $\mathrm{mm}$} \\
\hline Median (Q1-Q3) & $2.8(2.2-3.2)$ & $2(1.8-2.4)$ & $0.004^{\mathrm{a}, * *}$ \\
\hline \multicolumn{4}{|c|}{ Left olfactory cleft width, $\mathrm{mm}$} \\
\hline Median (Q1-Q3) & $2.75(2.3-3.1)$ & $2.05(1.8-2.35)$ & $\mathbf{0 . 0 0 5 ^ { a , * * }}$ \\
\hline \multicolumn{4}{|c|}{ Olfactory cleft volume, $\mathrm{cm}^{3}$} \\
\hline Median (Q1-Q3) & $325.5(243-367)$ & $169(155-210)$ & $0.003^{\mathrm{a}, * *}$ \\
\hline \multicolumn{4}{|l|}{ Threshold } \\
\hline Median (Q1-Q3) & $1(1-2.5)$ & $12(6.5-13)$ & $0.002^{\mathrm{a}, * *}$ \\
\hline \multicolumn{4}{|l|}{ Discrimination } \\
\hline \multicolumn{4}{|l|}{ Identification } \\
\hline Median (Q1-Q3) & $2(0-5)$ & $12(8.5-13)$ & $0.013^{a, *}$ \\
\hline \multicolumn{4}{|l|}{ TDI total } \\
\hline Median (Q1-Q3) & $4(1-9.5)$ & $37(21.5-39)$ & $0.004^{\mathrm{a}, * *}$ \\
\hline $\begin{array}{l}{ }^{a} \text { Mann-Whitney } \\
\text { TDI, threshold-dis } \\
{ }^{*} p<0.05 \text {. } \\
{ }^{* *} p<0.01 \text {. }\end{array}$ & & & \\
\hline
\end{tabular}

When both Group 1 and 2 cases were included, there was a significant negative correlation between total TDI scores and olfactory cleft widths and total volumes $(r=-0.665 ; r=$ -0.731 , respectively) (Table 3; Fig. 3, 4). Backward regression analysis was performed to assess the effect of age, right cleft width, left cleft width, and cleft volume on TDI. Analysis showed a significant model $(F=5.318 ; p<0.05)$ with an R2 of 0.195 . The effect of age, right and left cleft width, and cleft volume on TDI value was $19.5 \%$. As a result of the analysis, the study ended in step 4; cleft volume was found to

Olfactory Cleft Width and Volumes in Patients with COVID-19 have a significant effect on TDI in the model $(p=0.031)$ (Table 4). The flowchart summarizing the patients' olfactory tests and radiological findings is demonstrated in Figure 5.

\section{Discussion}

Olfactory clefts constitute the narrow, upper parts of nasal cavities located between the nasal septum and medial turbinate and are lined with olfactory epithelium [23, 


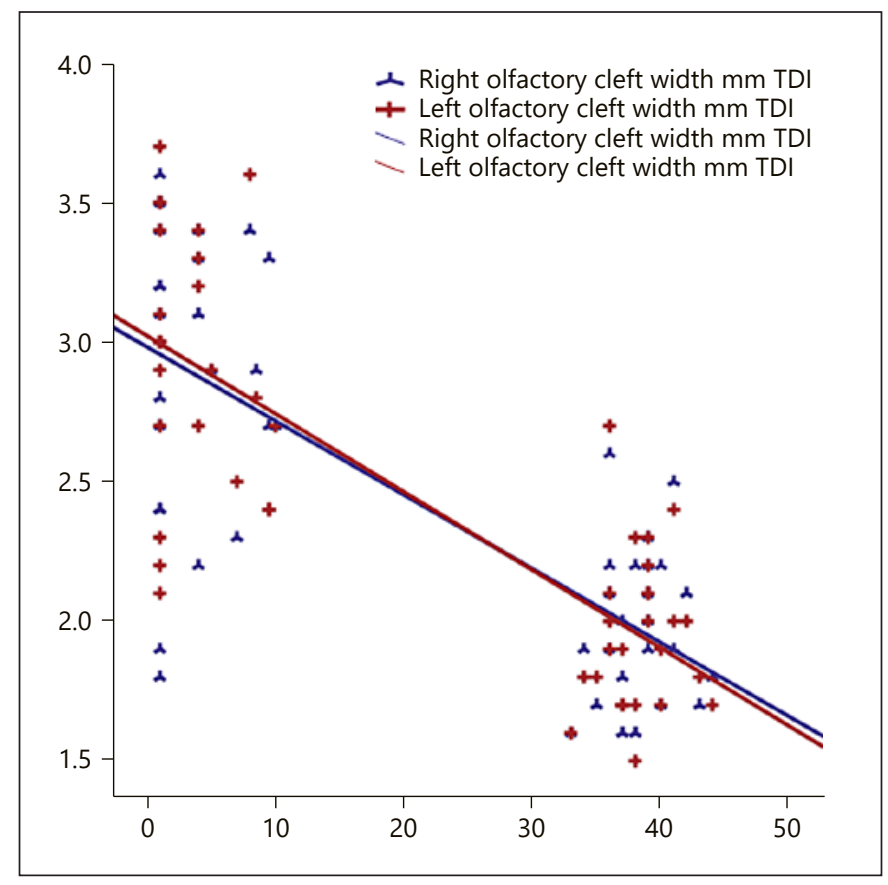

Fig. 3. The relation between right/left olfactory cleft widths and TDI. TDI, threshold-discrimination-identification.

Table 3. Relation between right/left olfactory cleft widths, olfactory cleft volume and TDI in COVID-19 patients grouped based on the presence of OD

\begin{tabular}{ll}
\hline & $\begin{array}{l}\text { TDI } \\
\text { Total }\end{array}$ \\
\hline $\begin{array}{ll}\text { Olfactory cleft width, } \mathrm{mm} \\
r\end{array}$ & \\
$\quad p$ value & -0.665 \\
Olfactory cleft volume, $\mathrm{cm} 3$ & $\mathbf{0 . 0 0 1 * *}$ \\
$\quad r$ & \\
$p$ value & -0.731 \\
\hline
\end{tabular}

$r=$ Spearman's Correlation Coefficient.

OD, olfactory dysfunction; TDI, threshold-discriminationidentification.

** $p<0.01$.

24]. Odorants in the nasal cavity reach the olfactory cleft and initiate a cascade of G-protein mediated reactions in the bipolar olfactory neurons. Olfactory nerve fibers originating from olfactory neurons cross the cribriform plate and synapse in the olfactory bulbs and are subsequently relayed to the primary or secondary olfactory cortex [24]. Olfactory mucosa is composed of olfactory

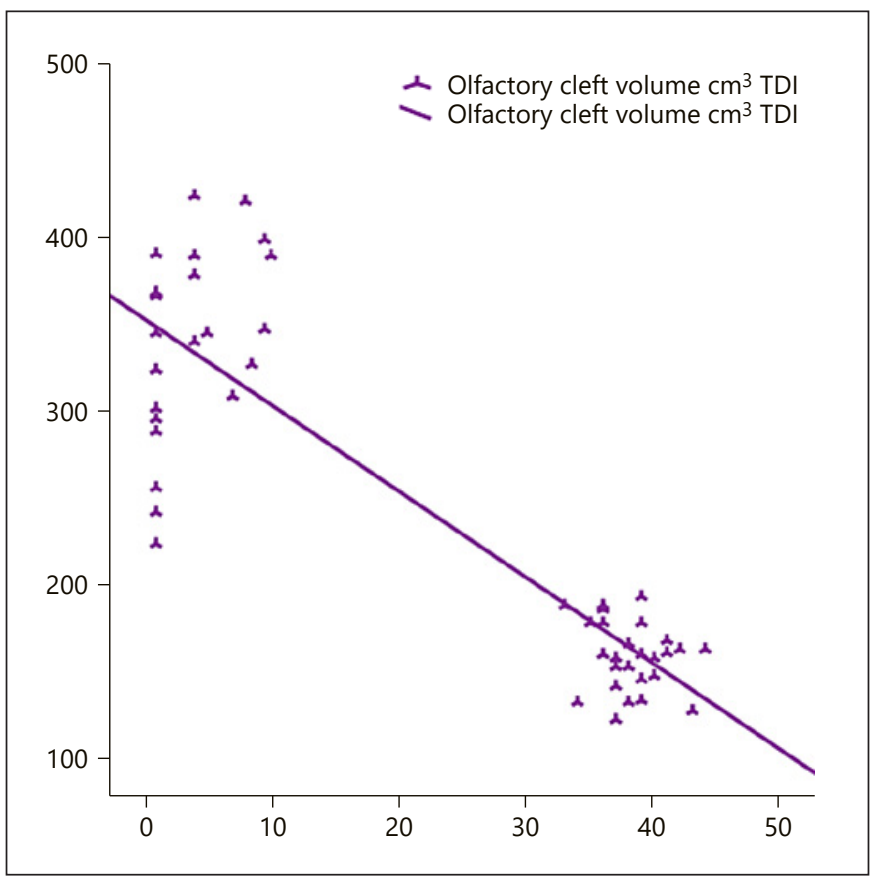

Fig. 4. There is a significant negative relation between olfactory cleft volume and TDI in the whole study group. TDI, thresholddiscrimination-identification.

neurons, sustentacular cells, and epithelial cells. Different hypotheses have been proposed for SARS-CoV-2-related anosmia including direct epithelial and olfactory receptor injury, changes secondary to inflammation related to nasal cytokine storm, and central neuropathic effects. The most widely accepted theory is direct olfactory epithelial and receptor injury due to viral invasion $[25,26]$. According to the general hypothesis, as the olfactory cleft width increases, the mucosal surface area also increases. Viral damage will be more pronounced as the width of the mucosa exposed to the virus increases [12, 14, 27-29]. Also patients with neurodegenerative disease, chronic rhinosinusitis, and chronic irritant/pollutant exposure have OD related to olfactory mucosal damage too [24-26].

SARS-CoV-2 targets the angiotensin-converting enzyme receptor-2 (ACE2) and transmembrane protease serine 2 for cell entry and inflammatory response [30]. The respiratory epithelium has a high level of expression of these receptors, underlying the predominance of respiratory symptoms [30]. SARS-CoV-2's tropism for olfactory epithelium is also related to the high level of ACE2 receptor expression in olfactory epithelium [26]. Studies have demonstrated the direct cellular injury of the virus via these receptors. Anosmia might be related to direct neuropathic injury by the virus; however, recent studies 
Fig. 5. Flowchart summarizing odor tests and radiological findings of patients.

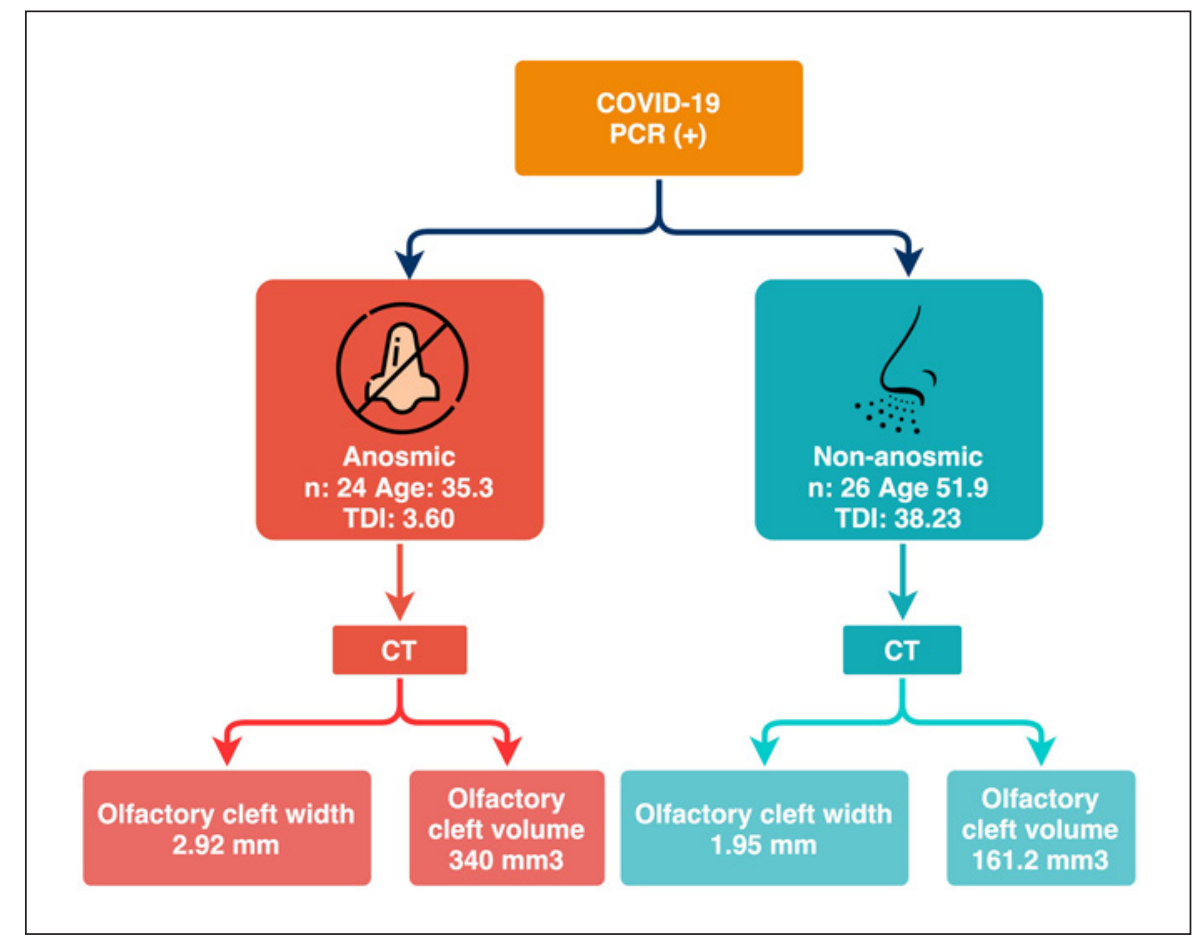

Table 4. Regression analysis of the effect of age, right cleft width, left cleft width, and cleft volume on TDI in patients with anosmia

\begin{tabular}{|c|c|c|c|c|}
\hline \multirow[t]{2}{*}{ Model } & \multirow{2}{*}{$\begin{array}{l}\text { Unstandardized coefficients } \\
B\end{array}$} & \multirow[t]{2}{*}{$p$ value } & \multicolumn{2}{|l|}{$95.0 \% \mathrm{Cl}$ for $\mathrm{B}$} \\
\hline & & & lower bound & upper bound \\
\hline 4 Cleft volume & 0.028 & $0.031 *$ & 0.003 & 0.053 \\
\hline
\end{tabular}

$\mathrm{Cl}$, confidence interval; TDI, threshold-discrimination-identification.

Dependent variable: TDI. $* p<0.05$ have shown that ACE2 receptor expression in olfactory mucosa is more common in sustentacular and supplementary cells rather than the olfactory receptor or epithelial cells. OD might actually be related to the supplementary cell damage [31-34]. Direct nasal mucosal damage and mucosal/epithelial damage secondary to inflammation may hinder the transfer of odorants to olfactory receptor cells; additionally, supplementary cell damage may result in the insufficiency of blood supply and regeneration resulting in olfactory hypofunction.

Similar to chronic rhinosinusitis and post-viral anosmia, a high level of cytokine release mediated by TNF- $\alpha$ and IL- $1 \beta$ has been shown in early onset COVID-19 anosmia $[29,35]$. An increase in viral load leads to a higher grade of inflammation and damage, resulting in a higher degree of OD [36]. In this aspect, an increase in olfactory

Olfactory Cleft Width and Volumes in Patients with COVID-19 cleft width and olfactory volume may lead to an overall higher ACE2 and transmembrane protease serine 2 receptor expression and mucosal surface for virus attachment. This subsequently may increase direct mucosal or "nasal cytokine storm" related damage resulting in a higher risk of OD. In a recent study conducted by our research group, we showed that the width and volume of the olfactory cleft were larger in cases with postinfectious/ post-viral anosmia than in the control group [27]. We conducted a similar olfactory cleft morphology study with the idea that the same situation may be valid for the SARS-CoV-2 virus. Similar to the results of postinfectious anosmia study, we showed larger olfactory cleft widths and volumes in COVID-19 anosmia in this study. We think that this might be an underlying predisposing factor, facilitating epithelial and receptor damage secondary 
to increased olfactory mucosal surface area and higher mucosal viral exposure.

Studies so far have shown that viruses may affect vital structures like the heart and brain resulting in fatal complications like cardiomyopathy, heart failure, arrhythmia, hemorrhagic encephalitis, and stroke [37-39]. Hematogenous spread from respiratory epithelium to these structures is a plausible mechanism; however, central nervous system involvement might be related to direct intracranial spread via the olfactory receptor-olfactory bulb pathway as shown in some other viruses $[31,40,41]$. In this aspect, olfactory cleft-volume relation to virus contact area, nasal cytokine storm, and neurologic involvement raise an important question.

In a study of Worley et al. [42] they showed age-associated differences in sinonasal anatomy, increase in olfactory volume, and decrease in olfactory function with age, more pronounced in ages $>40$. It was hypothesized that increased volume could be related to bony contraction around the intranasal space with additional atrophic changes in nasal and turbinate mucosa. Changes in sympathetic/parasympathetic innervation to the nasal cavity may result in decrease in nasal cycle patterns with age. OD might be related to alteration of intranasal heat and humidity exchange due to changing airflow patterns. In our study, patients in Group 2 had a higher mean age than that in Group 1. Based on Worley et al. [42] study, olfactory cleft volume should increase with aging and theoretically Group 2 patients should have had higher olfactory cleft volumes. However, in our study, patients in Group 2 (cases without COVID-19 anosmia) had lower olfactory cleft volumes. This situation shows us that the COVID-19 associated anosmia is associated with other factors than age.

Limitations of the current study include relatively limited patient number in each study group. COVID-19 anosmia cases did not have baseline CTs available prior to the infection to demonstrate the changes in the olfactory cleft in the infection course and assess whether this findings represent a predisposing factor or sequela of the infection course. Additionally, patients in the second group had imaging done prior to COVID-19 infection. Constituting a control group was difficult for this study. Patients without COVID-19 anosmia could not be scanned with paranasal sinus CT due to cost, radiation exposure, and ethical issues. For this reason, we did a retrospective chart/imaging review to identify cases that had prior paranasal sinus CTs over a 2-year interval. We selected cases that underwent CT for indications like tinnitus or headache that had not been shown to have a relation to olfactory cleft or olfactory function so far. We have excluded patients with nasal septal deviation or pathologically hypertrophied concha since such findings could affect the airflow dynamics in the nasal cavity. We did not repeat paranasal sinus imaging after COVID-19 infection due to ethical concerns.

Another detail about the study was that some of the patients may not notice their symptoms and recover naturally during waiting evaluation of Sniffin' Sticks. This situation can be considered among the limitations of the study.

In conclusion, this study had shown increased olfactory cleft width/volume in cases with COVID-19 anosmia compared to COVID-19 cases without OD. Total TDI score was found to be inversely correlated with cleft width and volume. These findings suggest that increased olfactory cleft size may be a predisposing factor for COVID-19 anosmia. Although this is accepted as a hypothesis according to our study's results, we think that it can be proven in the future with new studies designed using the latest technological software programs.

\section{Statement of Ethics}

The study protocol was approved by the Medical Research Ethics Committee of Acibadem University (decision No: 2020-13/6, date. June 25,2020 ). The study protocol conforms to the ethical guidelines of the 1975 Declaration of Helsinki as reflected in a priori approval by the institution's human research committee. Patients were not required to give their informed consent for inclusion in this retrospective study because we used anonymous clinical data and individual cannot be identified according to the data present.

\section{Conflict of Interest Statement}

The authors declare that there is no conflict of interest.

\section{Funding Sources}

The authors received no financial support for the research, authorship, and/or publication of this article.

\section{Author Contributions}

D.E.T.S. contributed to investigation, data curation, writing the original draft, review, and editing; A.A. contributed to conceptualization, methodology, validation, formal analysis, review, and editing; D.Y. contributed to writing - the original draft, review, and editing, S.G.K. contributed to data curation, review, and editing; and A.N.S. contributed to data curation, review, and editing.
Tekcan Sanli/Altundag/Yıldırım/ Kandemirli/Sanli 


\section{References}

1 Parma V, Ohla K, Veldhuizen MG, Niv MY, Kelly CE, Bakke AJ, et al. More than smellCOVID-19 is associated with severe impairment of smell, taste, and chemesthesis. Chem Senses. 2020;45(7):609-22.

2 Bagheri SH, Asghari A, Farhadi M, Shamshiri AR, Kabir A, Kamrava SK, et al. Coincidence of COVID-19 epidemic and olfactory dysfunction outbreak in Iran. Med J Islam Repub Iran. 2020;34(62);446-52.

3 Gane SB, Kelly C, Hopkins C. Isolated sudden onset anosmia in COVID19 infection. A novel syndrome? Rhinology. 2020;58(3):299-301.

4 Giacomelli A, Pezzati L, Conti F, Bernacchia D, Siano M, Oreni L, et al. Self-reported olfactory and taste disorders in patients with severe acute respiratory coronavirus 2 infection: a cross-sectional study, Clin Infect Dis. 2020; 71(15):889-90.

5 Vroegop AV, Eeckels AS, Van Rompaey V, Abeele VD, Schiappoli M, Alobid I, et al. COVID-19 and olfactory dysfunction - an ENT perspective to the current COVID-19 pandemic. B-ENT. 2020;16(1):81-5

6 Çetinkaya EA, Selçuk ÖT, Eyigör H, Gür ÖE. COVID-19 and anosmia. ENT-HNS. 2020; 28(Suppl 1):S61-S63

7 Hjelmesæth J, Skaare D. Loss of smell or taste as the only symptom of COVID-19. Tidsskr Nor Laegeforen. 2020;140:7.

8 Heidari F, Karimi E, Firouzifar M, Khamushian P, Ansari R, Mohammadi Ardehali M, et al. Anosmia as a prominent symptom of COVID-19 infection. Rhinology. 2020;58(3): 302-3

9 Hopkins C, Surda P, Kumar N. Presentation of new onset anosmia during the COVID-19 pandemic. Rhinology. 2020;58(3):295-8.

10 Lechien JR, Cabaraux P, Chiesa-Estomba CM, Khalife M, Plzak J, Hans S, et al. Objective olfactory testing in patients presenting with sudden onset olfactory dysfunction as the first manifestation of confirmed COVID-19 infection. MedRxiv. 2020.

11 Yan CH, Faraji F, Prajapati DP, Boone CE DeConde AS. Association of chemosensory dysfunction and COVID-19 in patients presenting with influenza-like symptoms. Int Forum Allergy Rhinol. 2020;10(7):806-13.

12 Yildirim D, Altundag A, Tekcan Sanli DE, Bakir A, Eryurekli A, Alis D, et al. A new perspective on imaging of olfactory dysfunction does size matter? Eur J Radiol. 2020;132:109290.

13 Goncalves S, Goldstein BJ. Pathophysiology of olfactory disorders and potential treatment strategies. Curr Otorhinolaryngol Rep. 2020; 4(2):115-21.

14 Altundag A, Yildırım D, Tekcan Sanli DE, Cayonu M, Kandemirli SD, Necati Sanli A, et al. Olfactory cleft measurements and COVID-19-related anosmia. Otolaryngol Head Neck Surg. 2020

15 Mitrano DA, Houle SE, Pearce P, Quintanilla RM, Lockhart BK, Genovese BC, et al. Olfactorydysfunction in the $3 \mathrm{xTg}-\mathrm{AD}$ model of
Alzheimer's disease. IBRO NeurosciRep. 2020;10:51-61.

16 Zou YM, Lu D, Liu LP, Zhang HH, Zhou YY. Olfactory dysfunction in Alzheimer's disease. Neuropsychiatr Dis Treat. 2016;12:869-75.

17 Hummel T, Sekinger B, Wolf SR, Pauli E, Kobal G. Sniffin' sticks': olfactory performance assessed by the combined testing of odor identification, odor discrimination and olfactory threshold. Chem Senses. 1997;22(1):39_ 52.

18 Kobal G, Klimek L, Wolfensberger M, Gudziol H, Temmel A, Owen CM, et al. Multicenter investigation of 1,036 subjects using a standardized method for the assessment of olfactory function combining tests of odor identification, odor discrimination, and olfactory thresholds. Eur Arch Otorhinolaryngol. 2000; 257(4):205-11.

19 Wolfensberger M, Schnieper I, Welge-Lüssen A. Sniffin' sticks: a new olfactory test battery. Acta Otolaryngol. 2000;120(2):303-6.

20 Hummel T, Kobal G, Gudziol H, Mackay-Sim A. Normative data for the "Sniffin' sticks": including tests of odor identification, odor discrimination, and olfactory thresholds: an upgrade based on a group of more than 3,000 subjects. Eur Arch Otorhinolaryngol. 2007; 264(3):237-43.

21 Tekeli H, Altundağ A, Salihoğlu M, Cayönü M, Kendirli MT. The applicability of the "Sniffin' sticks" olfactory test in a Turkish population. Med Sci Monit. 2013;19:1221-6.

22 Worley ML, Schlosser RJ, Soler ZM, Dubno JR, Eckert MA. Age-related differences in olfactory cleft volume in adults: a computational volumetric study. Laryngoscope. 2020; 129(2):E55-60.

23 Holbrook EH, Wu E, Curry WT, Lin DT, Schwob JE. Immunohistochemical characterization of human olfactory tissue. Laryngoscope. 2011;121(8):1687-701.

24 Hummel T, Whitcroft KL, Andrews P, Altundag A, Cinghi C, Costanzo RM, et al. Position paper on olfactory dysfunction. Rhinol Suppl. 2017;54(26):1-30.

25 Hawkes C. Olfaction in neurodegenerative disorder. Adv Otorhinolaryngol. 2006;63: 133-51.

26 Ajmani GS, Suh HH, Pinto JM. Effects of ambient air pollution exposure on olfaction: a review. Environ Health Perspect. 2016;124(11): 1683-93.

27 Altundag A, Temirbekov D, Haci C, Yildirim D, Cayonu M. Olfactory cleft width and volume: possible risk factors for postinfectious olfactory dysfunction. Laryngoscope. 2021; 131(1):5-9.

28 Sanli DET, Altundag A, Kandemirli SG, Yildirim D, Sanli AN, Saatci O, et al. Relationship between disease severity and serum IL-6 levels in COVID-19 anosmia. Am J Otolaryngol. 2021 Jan-Feb;42(1):102796.

29 Torabi A, Mohammadbagheri E, Akbari Dilmaghani N, Bayat AH, Fathi M, Vakili K, et al. Proinflammatory cytokines in the olfactory mucosa result in COVID-19 induced anosmia. ACS Chem Neurosci. 2020;11(13): 1909-13.

30 Yazdanpanah N, Saghazadeh A, Rezaei N. Anosmia: a missing link in the neuroimmunology of coronavirus disease 2019 (COVID-19). Rev Neurosci. 2020;31(7):691-701.

31 Baig AM, Khaleeq A, Ali U, Syeda H. Evidence of the COVID-19 virus targeting the CNS: tissue distribution, host-virus interaction, and proposed neurotropic mechanisms. ACS Chem Neurosci. 2020;11(7):995-8.

32 Bilinska K, Jakubowska P, Von Bartheld CS, Butowt R. Expression of the SARS-CoV-2 entry proteins, ACE2 and TMPRSS2, in cells of the olfactory epithelium: identification of cell types and trends with age. ACS Chem Neurosci. 2020;11(11):1555-62.

33 Brann DH, Tsukahara T, Weinreb C, Lipovsek M, Van den Berge K, Gong B, et al. Non-neuronal expression of SARS-CoV-2 entry genes in the olfactory system suggests mechanisms underlying COVID-19-associated anosmia. Sci Adv. 2020;6(31):eabc5801.

34 Fodoulian L, Tuberosa J, Rossier D, Landis $\mathrm{BN}$, Carleton A, Rodriguez I. SARS-CoV-2 receptor and entry genes are expressed by sustentacular cells in the human olfactory neuroepithelium. BioRxiv. 2020.

35 Sánchez-Vallecillo MV, Fraire ME, BaenaCagnani C, Zernotti ME. Olfactory dysfunction in patients with chronic rhinosinusitis. Int J Otolaryngol. 2012;2012(2012):327206.

36 Meng X, Deng Y, Dai Z, Meng Z. COVID-19 and anosmia: a review based on up-to-date knowledge. Am J Otolaryngol. 2020;41(5): 102581 .

37 Long B, Brady WJ, Koyfman A, Gottlieb M. Cardiovascular complications in COVID-19. Am J Emerg Med. 2020;38(7):1504-7.

38 Carod-Artal FJ. Neurological complications of coronavirus and COVID-19. Rev Neurol. 2020;70(9):311-22

39 Kandemirli SG, Dogan L, Sarikaya ZT, Kara S, Akinci C, Kaya D, et al. Brain MRI findings in patients in the intensive care unit with COVID-19 infection. Radiology. 2020;297(1): E232-5.

40 Ylikoski J, Markkanen M, Mäkitie A. Pathophysiology of the COVID-19 - entry to the CNS through the nose. Acta Otolaryngol. 2020;140(10):886-9.

41 Lechien JR, Chiesa-Estomba CM, De Siati DR, Horoi M, Le Bon SD, Rodriguez A, et al. Olfactory and gustatory dysfunctions as a clinical presentation of mild-to-moderate forms of the coronavirus disease (COVID-19): a multicenter European study. Eur Arch Otorhinolaryngol. 2020;277(8):2251-61.

42 Worley ML, Schlosser RJ, Soler ZM, Dubno JR, Eckert MA. Age-relateddifferences in olfactorycleftvolume in adults: a computational volumetric study. Laryngoscope. 2019;129(2): E55-E60.
Olfactory Cleft Width and Volumes in Patients with COVID-19
ORL 2022:84:1-9 